\title{
Fundamental Frequency Analysis of Sandwich Beams with Functionally Graded Face and Metallic Foam Core
}

\author{
Lin Mu and Guiping Zhao \\ State Key Laboratory of Mechanical Structure Strength and Vibration, School of Aerospace, Xi'an Jiaotong University, \\ Xian 710049, China \\ Correspondence should be addressed to Guiping Zhao; zhaogp@mail.xjtu.edu.cn
}

Received 5 August 2015; Revised 7 November 2015; Accepted 11 November 2015

Academic Editor: Jussi Sopanen

Copyright (C) 2016 L. Mu and G. Zhao. This is an open access article distributed under the Creative Commons Attribution License, which permits unrestricted use, distribution, and reproduction in any medium, provided the original work is properly cited.

\begin{abstract}
This study is interested in assessing a way to analyze fundamental frequency of sandwich beams with functionally graded face sheet and homogeneous core. The face sheet, which is an exponentially graded material (EGM) varying smoothly in the thickness direction only, is composed of a mixture of metal and ceramic. The core which is made of foam metal is homogeneous. The classical plate theory (CPT) is used to analyze the face sheet and a higher-order theory (HOT) is used to analyze the core of sandwich beams, in which both the transverse normal and shear strains of the core are considered. The extended Galerkin method is used to solve the governing equations to obtain the vibration equations of the sandwich beams suitable for numerical analysis. The fundamental frequency obtained by the theoretical model is validated by using the finite element code ABAQUS and comparison with earlier works. The influences of material and geometric properties on the fundamental frequency of the sandwich beams are analyzed.
\end{abstract}

\section{Introduction}

Functionally graded materials (FGM), consisting of two or more constituent phases with continuous and smoothly varying composition, are one of advanced materials in the family of engineering composites [1]. FGM refers to a heterogeneous composite material with gradient variation from one surface of the material to the other which results in continuously varying material properties [2]. Compared with fiberreinforced composite laminates, FGM can eliminate the stress concentration at the interface of the layers and overcome the delaminating in composite laminates [3]. Functionally gradient ceramic materials are usually made from a mixture of metal and ceramics. In the mixture, the ceramic can resist high temperature in thermal environments, while the metal can decrease the tensile stress on the ceramic surface at the earlier state of cooling. These FGM are widely used in great application potential in many engineering sectors such as aerospace vehicles and power generators.

Many researchers have investigated the static and the dynamic characteristics of functionally graded sandwich structures. Zenkour $[4,5]$ presented a two-dimensional solution for bending analysis of simply supported functionally graded ceramic-metal sandwich plate and studied the bucking and free vibration of the simply supported functionally graded sandwich plate. In his study, the faces of sandwich plate are made of functionally graded material, and the core layer is made of the isotropic ceramic material. Woodward and Kashtalyan [6] presented a 3D elasticity solution for bending response of sandwich plate, in which the stiffness of the core varies gradually in the thickness direction. The use of graded core to improve performance of sandwich structures, especially under localized loading, is examined and discussed. Zenkour and Alghamdi [7] studied the bending issue of sandwich plate with functionally graded faces and homogeneous ceramic core under mechanical and thermal loads. Cinefra and Soave [8] presented the closed form solutions for free vibration of simply supported sandwich plate with isotropic faces and functionally graded core, which are composed of two different materials. Kashtalyan and Menshykova [9] presented a three-dimensional elasticity solution for sandwich plate with a functionally graded core subjected to transverse loading. Their studies indicated that the use of graded core instead of a conventional homogeneous one can eliminate discontinuity of the in-plane normal and shear stresses across the adjoining layers (between 
the face sheets and the core). Based on the three-dimensional elastic theory, $\mathrm{Li}$ et al. [10] studied the free vibration of functionally graded sandwich plates with simply supported and clamped edges. Neves et al. [11, 12] analyzed the free vibration of sandwich plate/shell with gradient core using the Carrera Unified Formulation (CUF) $[13,14]$ and meshless method [15]. Thai et al. [16] presented a new first-order shear deformation theory for functionally graded sandwich plates composed of functionally graded face sheets and an isotropic homogeneous core. And analytical solutions for bending, buckling, and free vibration analysis of rectangular plates under various boundary conditions are presented. Based on a refined shear deformation theory, Vo et al. [17] presented a finite element model for vibration and buckling of functionally graded sandwich beams, which is made of metallic or ceramic core and functional graded face sheets.

Although many researchers have investigated the mechanical properties of the functionally graded sandwich structures, the vibration characteristics of the sandwich structures with graded face sheets and metallic foam cores have been of little concern up to now. And the sandwich structures with graded face sheets and metallic foam core were widely used for the leading edges of the swing on airplane or submarine hull, which require high specific stiffness and specific strength.

In this study, we develop a way to analyze composite sandwich beams consisting of graded face sheets and metallic foam core, which is very different from metallic or ceramic core that has been analyzed in literature. The face sheets of the sandwich beams are a nonhomogeneous composite which feature a gradual change in properties with the continuous change of component (ceramic-metal) from one surface to the other. Young's modulus and density are characterized by a power law distribution. The core of the sandwich beam is isotropic aluminum foams. The fundamental frequency of the sandwich beams is calculated and influences of material and geometric properties on the fundamental frequency of the sandwich beams are analyzed.

\section{Mechanical Model}

The mechanical model is described in Figure 1. In this model, $t^{f}$ is the thickness of face sheet and $t^{c}$ measures the thickness of the core. $L$ is the length of the sandwich beam and $H$ is the thickness of the sandwich beam. In the local Cartesian coordinate system $(x, z), x$ - and $z$-axis are set to the centerline of global mid-surface of the beam and thickness direction, respectively. So the global mid-surface is the beam reference surface.

The basic assumptions of the model are as follows:

(1) The face sheets are composed of functionally graded materials through thickness direction, Young's modulus and material densities vary continuously in thickness direction, and Poisson's ratio is assumed constant.

(2) The face sheets are very thin so that they can carry the tangential and bending loads and follow Kirchhoff hypothesis.

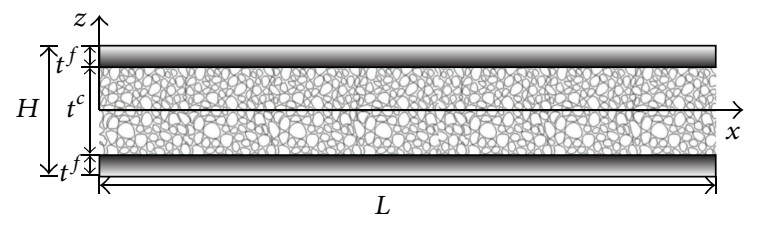

FIGURE 1: Functionally graded sandwich beam.

(3) The core is made of isotropic soft material and capable of carrying transverse normal and shear stresses.

(4) The deflection in transverse direction is large, while the tangential deformation of beam is small as the tangential stiffness of the sandwich beam is large. In the process of deformation, the face sheets are considered to be incompressible in the transverse direction while the core, due to its soft material, is considered to be compressible.

2.1. Displacements of the Model. The displacements of the sandwich beam can be properly described by $v_{1}^{t}$ and $v_{3}^{t}$ in $x$ and $z$ directions of the top face sheet, respectively:

$$
\begin{aligned}
& v_{1}^{t}=u_{1}^{t}(x, t)-\left(z-\frac{t^{c}+t^{f}}{2}\right) u_{3,1}^{t}(x, t) \\
& v_{3}^{t}=u_{3}^{t}(x, t),
\end{aligned}
$$

where $u_{1}^{t}(x, t)$ and $u_{3}^{t}(x, t)$ are the mid-surface displacements in $x$ and $z$ directions of the top face sheet, respectively. By analogy, $v_{1}^{b}$ and $v_{3}^{b}$ are the displacements in $x$ and $z$ directions of the bottom face sheet, respectively:

$$
\begin{aligned}
& v_{1}^{b}=u_{1}^{b}(x, t)-\left(z+\frac{t^{c}+t^{f}}{2}\right) u_{3,1}^{b}(x, t) \\
& v_{3}^{b}=u_{3}^{b}(x, t),
\end{aligned}
$$

where $u_{1}^{b}(x, t)$ and $u_{3}^{b}(x, t)$ are the mid-surface displacements in $x$ and $z$ directions of the bottom face sheet, respectively.

For the core, $v_{1}^{c}$ and $v_{3}^{c}$ represent the displacements in $x$ and $z$ directions of the core, respectively. The tangential displacement is expanded in a second-order power series to the thickness direction; only a first-order term is used for the transverse displacement [18]. Consider

$$
\begin{aligned}
v_{1}^{c}= & u_{1}^{a}(x, t)+\frac{t^{f}}{2} u_{3,1}^{d}(x, t)+\frac{2 z}{t^{c}} u_{1}^{d}(x, t) \\
& +\frac{t^{f} z}{t^{c}} u_{3,1}^{a}(x, t)+\left[\frac{4 z^{2}}{\left(t^{c}\right)^{2}}-1\right] \varphi_{1}^{c}(x, t) \\
v_{3}^{c}= & u_{3}^{a}(x, t)+\frac{2 z}{t^{c}} u_{3}^{d}(x, t),
\end{aligned}
$$


where $\varphi_{1}^{c}(x, t)$ is an additional warping function describing the deformation of the sandwich core. $u_{i}^{a}$ and $u_{i}^{d}(i=1,3)$ are defined as

$$
\begin{aligned}
& u_{i}^{a}=\frac{1}{2}\left(u_{i}^{t}+u_{i}^{b}\right) \\
& u_{i}^{d}=\frac{1}{2}\left(u_{i}^{t}-u_{i}^{b}\right) .
\end{aligned}
$$

2.2. Functionally Graded Materials. The face sheets of the sandwich beam are gradient composite made of ceramic $\left(\mathrm{AlO}_{2}\right)$ and metal (Al). Young's modulus and mass density vary continuously in the thickness direction $(z$-axis direction).

The effective material properties can be expressed as

$$
\begin{aligned}
& E(z) \\
& = \begin{cases}\left(E_{2}-E_{1}\right)\left(\frac{\left(t^{c} / 2+t^{f}-z\right)}{t^{f}}\right)^{p}+E_{1}, & z \in\left[\frac{t^{c}}{2}, \frac{t^{c}}{2}+t^{f}\right] \\
\left(E_{2}-E_{1}\right)\left(\frac{\left(t^{c} / 2+t^{f}+z\right)}{t^{f}}\right)^{p}+E_{1}, & z \in\left[-\frac{t^{c}}{2}-t^{f},-\frac{t^{c}}{2}\right],\end{cases}
\end{aligned}
$$

where $E_{1}, E_{2}$ are Young's modulus for the ceramic $\left(\mathrm{AlO}_{2}\right)$ and metal (Al), and

$$
\begin{aligned}
& \rho(z) \\
& = \begin{cases}\left(\rho_{2}-\rho_{1}\right)\left(\frac{\left(t^{c} / 2+t^{f}-z\right)}{t^{f}}\right)^{p}+\rho_{1}, & z \in\left[\frac{t^{c}}{2}, \frac{t^{c}}{2}+t^{f}\right] \\
\left(\rho_{2}-\rho_{1}\right)\left(\frac{\left(t^{c} / 2+t^{f}+z\right)}{t^{f}}\right)^{p}+\rho_{1}, & z \in\left[-\frac{t^{c}}{2}-t^{f},-\frac{t^{c}}{2}\right],\end{cases}
\end{aligned}
$$

where $\rho_{1}, \rho_{2}$ are the mass density for the ceramic $\left(\mathrm{AlO}_{2}\right)$ and metal (Al) and $p$ is the gradient exponent and $p \geq 0$.

2.3. Lagrangian of the Deformed System. The governing equations of sandwich beams are derived by Hamilton's principle of the minimization of the Lagrangian $L$ of the deformed system:

$$
\delta L=\int_{t_{1}}^{t_{2}}[\delta T-\delta U+\delta W] \mathrm{d} t=0,
$$

where $\delta T, \delta U$, and $\delta W$ denote the variations of the kinetic energy, strain energy, and external work performed during arbitrary instants of time $\left[t_{1}, t_{2}\right]$, respectively.

The variation of the kinetic energy and strain energy can be written as

$$
\begin{aligned}
\delta T & =\int_{0}^{l} \mathrm{~d} x\left(\int_{t^{c} / 2}^{t^{c} / 2+t^{f}} \rho^{f}(z)\left(\dot{v}_{1}^{t} \delta \dot{v}_{1}^{t}+\dot{v}_{3}^{t} \delta \dot{v}_{3}^{t}\right) \mathrm{d} z\right. \\
& +\int_{-t^{c} / 2}^{t^{c} / 2} \rho_{c} \dot{v}_{3}^{c} \delta \dot{v}_{3}^{c} \mathrm{~d} z \\
& \left.+\int_{-t^{c} / 2-t^{f}}^{-t^{c} / 2} \rho^{f}(z)\left(\dot{v}_{1}^{b} \delta \dot{v}_{1}^{b}+\dot{v}_{3}^{b} \delta \dot{v}_{3}^{b}\right) \mathrm{d} z\right)
\end{aligned}
$$

$$
\begin{gathered}
\delta U=\int_{0}^{l} \mathrm{~d} x \int_{t^{c} / 2}^{t^{c} / 2+t^{f}}\left(\sigma_{1}^{t} \delta \varepsilon_{1}^{t}\right) \mathrm{d} z+\int_{0}^{l} \mathrm{~d} x \\
\cdot \int_{-t^{c} / 2}^{t^{c} / 2}\left(\sigma_{3}^{c} \delta \varepsilon_{3}^{c}+\sigma_{13}^{c} \delta \varepsilon_{13}^{c}\right) \mathrm{d} z+\int_{0}^{l} \mathrm{~d} x \\
\cdot \int_{-t^{c} / 2-t^{f}}^{-t^{c} / 2}\left(\sigma_{1}^{b} \delta \varepsilon_{1}^{b}\right) \mathrm{d} z,
\end{gathered}
$$

where $\rho^{f}(z)$ is the mass density function of the face sheet, $\rho_{c}$ is the mass density of the core, and $\dot{v}_{i}^{t}, \dot{v}_{i}^{b}$, and $\dot{v}_{j}^{c}(i=1,3, j=3)$ denote the speeds of the top face sheet, bottom face sheet, and the core, respectively. In addition, it is very direct to express the variation of the work performed by external loads as

$$
\delta W=\int_{0}^{l}\left(q_{3}^{t} \delta v_{3}^{t}+q_{3}^{b} \delta v_{3}^{b}\right) \mathrm{d} x,
$$

where $q_{3}^{t}$ and $q_{3}^{b}$ are the uniformly distributed pressure on the top and bottom face sheets, respectively.

Substituting $\delta T, \delta U$, and $\delta W$ into Hamilton's principle in (7) and considering stress integrals through the thickness of the beam, the governing equations are obtained by setting the coefficients of $\delta u_{1}^{a}, \delta u_{1}^{d}, \delta u_{3}^{a}, \delta u_{3}^{d}$, and $\delta \varphi_{1}^{c}$ over the field of integration to zero separately:

$$
\begin{aligned}
& \delta u_{1}^{a}:-2 m_{0}^{f} \ddot{u}_{1}^{a}+2 m_{1}^{f} \ddot{u}_{3,1}^{a}+N_{1,1}^{t}+N_{1,1}^{b}=0 \\
& \delta u_{1}^{d}:-2 m_{0}^{f} \ddot{u}_{1}^{d}+2 m_{1}^{f} \ddot{u}_{3,1}^{d}+N_{1,1}^{t}-N_{1,1}^{b}-\frac{2 N_{13}^{c}}{t^{c}}=0 \\
& \delta u_{3}^{a}:-\left(2 m_{0}^{f}+m_{0}^{c}\right) \ddot{u}_{3}^{a}-2 m_{1}^{f} \ddot{u}_{1,1}^{a}+2 m_{2}^{f} \ddot{u}_{3,11}^{a} \\
& \quad+M_{1,11}^{t}+M_{1,11}^{b}+\left(1+\frac{t^{f}}{t^{c}}\right) N_{13,1}^{c}+q_{3}^{t}+q_{3}^{b}=0 \\
& \delta u_{3}^{d}:-\left(2 m_{0}^{f}+m_{1}^{c}\right) \ddot{u}_{3}^{d}-2 m_{1}^{f} \ddot{u}_{1,1}^{d}+2 m_{2}^{f} \ddot{u}_{3,11}^{d} \\
& \quad+M_{1,11}^{t}-M_{1,11}^{b}-\left(\frac{2}{t^{c}}\right)\left(N_{3}^{c}-M_{13,1}^{c}\right)+q_{3}^{t} \\
& \quad-q_{3}^{b}=0 \\
& \delta \varphi_{1}^{c}: \frac{8}{\left(t^{c}\right)^{2}} M_{13}^{c}=0,
\end{aligned}
$$

where masses $m$ are given in Appendix A. The force resultants are given by

$$
\begin{aligned}
& N_{1}^{t}=\int_{t^{c} / 2}^{t^{c} / 2+t^{f}} \sigma_{1}^{t} \mathrm{~d} z \\
& M_{1}^{t}=\int_{t^{c} / 2}^{t^{c} / 2+t^{f}} \sigma_{1}^{t}\left(z-\frac{t^{c}+t^{f}}{2}\right) \mathrm{d} z \\
& N_{1}^{b}=\int_{-t^{c} / 2-t^{f}}^{-t^{c} / 2} \sigma_{1}^{b} \mathrm{~d} z
\end{aligned}
$$




$$
\begin{aligned}
M_{1}^{b} & =\int_{-t^{c} / 2-t^{f}}^{-t^{c} / 2} \sigma_{1}^{b}\left(z+\frac{t^{c}+t^{f}}{2}\right) \mathrm{d} z \\
N_{3}^{c} & =\int_{-t^{c} / 2}^{t^{c} / 2} \sigma_{3}^{c} \mathrm{~d} z \\
N_{13}^{c} & =\int_{-t^{c} / 2}^{t^{c} / 2} \sigma_{13}^{c} \mathrm{~d} z \\
M_{13}^{c} & =\int_{-t^{c} / 2}^{t^{c} / 2} \sigma_{13}^{c} z \mathrm{~d} z .
\end{aligned}
$$
mined

From (11a), (11b), (11c), (11d), (11e), and (11f) we deter-

$$
\begin{aligned}
& N_{1}^{t}=A_{1}^{t} u_{1,1}^{t}-B_{1}^{t} u_{3,11}^{t} \\
& M_{1}^{t}=B_{1}^{t} u_{1,1}^{t}-D_{1}^{t} u_{3,11}^{t} \\
& N_{1}^{b}=A_{1}^{b} u_{1,1}^{b}-B_{1}^{b} u_{3,11}^{b} \\
& M_{1}^{b}=B_{1}^{b} u_{1,1}^{b}-D_{1}^{b} u_{3,11}^{b},
\end{aligned}
$$

where $A_{1}^{t, b}, B_{1}^{t, b}$, and $D_{1}^{t, b}$ are given in Appendix B.

After substituting (12a), (12b), (12c), and (12d) into (10a), (10b), (10c), (10d), and (10e) we arrived at

$$
\begin{aligned}
& -\left(2 m_{0}^{f} \ddot{u}_{1}^{a}-2 m_{1}^{f} \ddot{u}_{3,1}^{a}\right)+2 A_{1}^{f} u_{1,11}^{a}-2 B_{1}^{f} u_{3,111}^{a}=0 \\
& -\left(2 m_{0}^{f} \ddot{u}_{1}^{d}-2 m_{1}^{f} \ddot{u}_{3,1}^{d}\right)+2 A_{1}^{f} u_{1,11}^{d}-2 B_{1}^{f} u_{3,111}^{d} \\
& -\frac{2 G^{c}}{t^{c}}\left[\left(t^{c}+t^{f}\right) u_{3,1}^{a}+2 u_{1}^{d}\right]=0 \\
& -\left(2 m_{0}^{f} \ddot{u}_{3}^{a}+2 m_{1}^{f} \ddot{u}_{1,1}^{a}-2 m_{2}^{f} \ddot{u}_{3,11}^{a}+m_{0}^{c} \ddot{u}_{3}^{a}\right) \\
& +2 B_{1}^{f} u_{1,111}^{a}-2 D_{1}^{f} u_{3,1111}^{d}+\left(1+\frac{t^{f}}{t^{c}}\right) \cdot G^{c} \\
& \quad \cdot\left[\left(t^{c}+t^{f}\right) u_{3,11}^{a}+2 u_{1,1}^{d}\right]+q_{3}^{t}+q_{3}^{b}=0 \\
& -\left(2 m_{0}^{f} \ddot{u}_{3}^{d}+2 m_{1}^{f} \ddot{u}_{1,1}^{d}-2 m_{2}^{f} \ddot{u}_{3,11}^{d}+m_{1}^{c} \ddot{u}_{3}^{d}\right) \\
& \quad+2 B_{1}^{f} u_{1,111}^{d}-2 D_{1}^{f} u_{3,1111}^{d}-\frac{4 E^{c}}{t^{c}} \cdot u_{3}^{d}+q_{3}^{t} \\
& \quad-q_{3}^{b}=0 .
\end{aligned}
$$

2.4. Extended Galerkin Method. Now $u_{1}^{a}, u_{1}^{d}, u_{3}^{a}$, and $u_{3}^{d}$ are cast into state-space form:

$$
\begin{aligned}
& u_{1}^{a} \approx \sum_{i=1}^{N s} \phi_{1 i}^{a}(x) q_{1 i}^{a}(t) \\
& u_{1}^{d} \approx \sum_{i=1}^{N s} \phi_{1 i}^{d}(x) q_{1 i}^{d}(t)
\end{aligned}
$$

$$
\begin{aligned}
& u_{3}^{a} \approx \sum_{i=1}^{N s} \phi_{3 i}^{a}(x) q_{3 i}^{a}(t) \\
& u_{3}^{d} \approx \sum_{i=1}^{N s} \phi_{3 i}^{d}(x) q_{3 i}^{d}(t) .
\end{aligned}
$$

At the same time, the boundary conditions of the sandwich beams are considered to be clamped at their two ends $(x=0$ and $l)$; then

$$
\begin{aligned}
u_{1}^{a}(0, t) & =u_{1}^{d}(0, t)=u_{3}^{a}(0, t)=u_{3}^{d}(0, t)=\frac{\partial u_{3}^{a}(0, t)}{\partial x} \\
& =\frac{\partial u_{3}^{d}(0, t)}{\partial x}=0 \\
u_{1}^{a}(l, t) & =u_{1}^{d}(l, t)=u_{3}^{a}(l, t)=u_{3}^{d}(l, t)=\frac{\partial u_{3}^{a}(l, t)}{\partial x} \\
& =\frac{\partial u_{3}^{d}(l, t)}{\partial x}=0
\end{aligned}
$$

with the initial conditions

$$
\begin{aligned}
& u_{1}^{a}(x, 0)=u_{1}^{d}(x, 0)=u_{3}^{a}(x, 0)=u_{3}^{d}(x, 0)=0 \\
& \dot{u}_{1}^{a}(x, 0)=\dot{u}_{1}^{d}(x, 0)=\dot{u}_{3}^{a}(x, 0)=\dot{u}_{3}^{d}(x, 0)=0 .
\end{aligned}
$$
taking

We could make the boundary conditions satisfied by

$$
\begin{aligned}
& \left\{\phi_{11}^{a}(x), \phi_{12}^{a}(x), \ldots, \phi_{1 N s}^{a}(x)\right\} \\
& \quad=\left\{\phi_{11}^{d}(x), \phi_{12}^{d}(x), \ldots, \phi_{1 N s}^{d}(x)\right\} \\
& \quad=\left\{\sin \pi \frac{x}{l}, \sin 2 \pi \frac{x}{l}, \ldots, \sin N s \pi \frac{x}{l}\right\} \\
& \left\{\phi_{31}^{a}(x), \phi_{32}^{a}(x), \ldots, \phi_{3 N s}^{a}(x)\right\} \\
& \quad=\left\{\phi_{31}^{d}(x), \phi_{32}^{d}(x), \ldots, \phi_{3 N s}^{d}(x)\right\} \\
& \quad=\left\{W_{1}(x), W_{2}(x), \ldots, W_{N s}(x)\right\},
\end{aligned}
$$

where $W_{i}(x)$ represents the $i$ th normalized eigenmode $(i=$ $1,2, \ldots, N s)$ of a clamped-clamped Euler-Bernoulli beam, defined as [19]

$$
W_{i}(x)=\frac{W_{i}^{0}(x)}{\sqrt{\int_{0}^{l}\left[W_{i}^{0}(x)\right]^{2} \mathrm{~d} x}}
$$

where $W_{i}^{0}(x)=\left[\sin \left(\beta_{i}(x / l)\right)-\sinh \left(\beta_{i}(x / l)\right)\right]-\left(\left(\sin \beta_{i}-\right.\right.$ $\left.\left.\sinh \beta_{i}\right) /\left(\cos \beta_{i}-\cosh \beta_{i}\right)\right)\left[\cos \left(\beta_{i}(x / l)\right)-\cosh \left(\beta_{i}(x / l)\right)\right]$ and $\beta_{i}$ is the positive $i$ th root of the equation $\cos \beta_{i} \cosh \beta_{i}-1=0$.

Solving (13a), (13b), (13c), and (13d) is tackled by using the extended Galerkin method and then the mass matrix and 


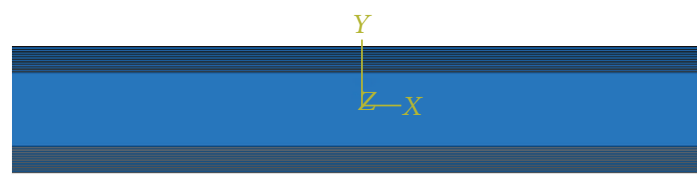

(a) The face sheets with ten layers

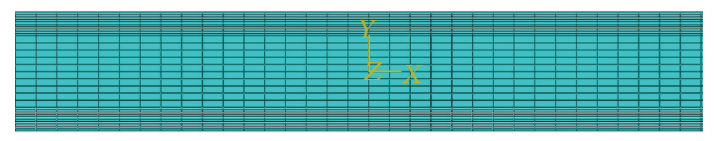

(b) FE model and mesh

Figure 2: Model of the sandwich beam.

stiffness matrix for the numerical analysis are determined as a matrix equation:

$$
\begin{gathered}
{\left[\begin{array}{cccc}
M_{11} & 0 & M_{13} & 0 \\
0 & M_{22} & 0 & M_{24} \\
M_{31} & 0 & M_{33} & 0 \\
0 & M_{42} & 0 & M_{44}
\end{array}\right]\left\{\begin{array}{l}
\left\{\ddot{q}_{1 i}^{a}\right\} \\
\left\{\ddot{q}_{1 i}^{d}\right\} \\
\left\{\ddot{q}_{3 i}^{a}\right\} \\
\left\{\ddot{q}_{3 i}^{d}\right\}
\end{array}\right\}} \\
+\left[\begin{array}{cccc}
K_{11} & 0 & K_{13} & 0 \\
0 & K_{22} & K_{23} & K_{24} \\
K_{31} & K_{32} & K_{33} & 0 \\
0 & K_{42} & 0 & K_{44}
\end{array}\right]\left\{\begin{array}{l}
\left\{q_{1 i}^{a}\right\} \\
\left\{q_{1 i}^{d}\right\} \\
\left\{q_{3 i}^{a}\right\} \\
\left\{q_{3 i}^{d}\right\}
\end{array}\right\}=\left\{\begin{array}{l}
0 \\
0 \\
f_{3} \\
f_{4}
\end{array}\right\} .
\end{gathered}
$$

The entities in mass matrix and stiffness matrix are listed in Appendix C.

\section{Validation of Gradient Sandwich Beam Model}

To assess the validity of the model of the sandwich beam with gradient face sheet, we calculate the natural frequencies and compare the results with those obtained by using ABAQUS code. The face sheet is divided into ten layers and the thickness of each layer is the same (see Figure 2(a)). Each layer of the face sheet model has 100 elements and the core model has 1000 elements. The sizes of meshes are $10 \mathrm{~mm} \times$ $1 \mathrm{~mm}$ for the face sheet and $10 \mathrm{~mm} \times 3 \mathrm{~mm}$ for the core. The CPE8R quadratic plane-strain elements are used for both core and face sheets. The nodes of the core and face sheets at their common interfaces are tied to ensure continuity of the displacements. Ends of the face sheets and core are taken to be clamped in the mode. The mesh of the sandwich beam is shown in Figure 2(b).

The material properties of the composite face sheets and the core are as follows.

The face sheets [3]:

$$
\begin{aligned}
E_{1} & =380 \mathrm{GPa}, \\
E_{2} & =70 \mathrm{GPa}, \\
\lambda & =0.3, \\
\rho_{1} & =3800 \mathrm{~kg} / \mathrm{m}^{3}, \\
\rho_{2} & =2707 \mathrm{~kg} / \mathrm{m}^{3}, \\
p & =1 .
\end{aligned}
$$

The core [20]:

$$
\begin{aligned}
& E_{c}=2.8 \mathrm{GPa}, \\
& \rho_{c}=150 \mathrm{~kg} / \mathrm{m}^{3} .
\end{aligned}
$$

Geometric parameters of the sandwich beam:

$$
\begin{aligned}
L & =1 \mathrm{~m}, \\
H & =0.05 \mathrm{~m}, \\
t^{f} & =0.01 \mathrm{~m}, \\
t^{c} & =0.03 \mathrm{~m}, \\
\frac{L}{H} & =20 .
\end{aligned}
$$

The first four frequencies of sandwich beam calculated by the present analytical model and numerical simulation are presented in Table $1 . K$ means the $K$ th natural frequency. For example, " $K=2$ " means the second-order natural frequency. It can be found that the natural frequencies of the gradient sandwich beam predicted by the analytical model are very close to those simulated by ABAQUS.

In order to further verify the present model, the fundamental frequencies of sandwich beams with different values of thickness ratio in comparison with earlier works [17] are given in Tables 2 and 3. $p$ means the gradient exponent and $\bar{w}$ means the nondimensional fundamental natural frequencies. It can be seen that the frequencies predicted by the present model do have an excellent agreement with these from Vo and Thai's model. Therefore, it can be concluded that the present analytical model is reliable and applicable.

\section{Result and Discussion}

In order to investigate the influences of material and geometric properties on the fundamental frequency of the sandwich beams, a sandwich beam of length $L=1 \mathrm{~m}$ and thickness $H=0.1 \mathrm{~m}$ is considered. The sandwich beam is composed of functionally graded face sheets and aluminum foam core. And boundary conditions are clamped. According to the researches by Ashby [21], the relationship of Young's modulus and density of closed-cell aluminum foam is as follows:

$$
E_{c}=\varphi\left[0.5\left(\frac{\rho_{c}}{\rho_{s}}\right)^{2}+0.3 \frac{\rho_{c}}{\rho_{s}}\right] E_{s} \quad(\varphi=0.1 \sim 1.0),
$$

where $E_{c}$ and $\rho_{c}$ are Young's modulus and mass density of closed-cell aluminum foam and $E_{s}$ and $\rho_{s}$ are Young's modulus and mass density of matrix material of the closed-cell 
TABLE 1: The first four frequencies $w(\mathrm{rad} / \mathrm{s})$ with increasing Ns (comparison between the present result and that of ABAQUS).

\begin{tabular}{|c|c|c|c|c|}
\hline \multirow{2}{*}{ Ns } & \multicolumn{4}{|c|}{$K$ th natural frequency $(\mathrm{rad} / \mathrm{s})$} \\
\hline & $K=1$ & $K=2$ & $K=3$ & $K=4$ \\
\hline 2 & $2416.0(3.46 \%)$ & $6050.6(20.12 \%)$ & - & - \\
\hline 3 & $2389.5(2.33 \%)$ & $5248.6(4.20 \%)$ & $9200.2(12.20 \%)$ & - \\
\hline 4 & $2371.8(1.57 \%)$ & $5185.0(2.94 \%)$ & $8471.7(3.32 \%)$ & $12544.5(8.64 \%)$ \\
\hline 5 & $2358.4(0.99 \%)$ & $5152.6(2.29 \%)$ & $8403.1(2.48 \%)$ & $11832.7(2.47 \%)$ \\
\hline 6 & $2354.7(0.84 \%)$ & $5119.5(1.64 \%)$ & $8365.6(2.02 \%)$ & $11773.4(1.96 \%)$ \\
\hline 7 & $2348.5(0.57 \%)$ & $5110.7(1.46 \%)$ & $8328.5(1.57 \%)$ & $11732.7(1.61 \%)$ \\
\hline 8 & $2347.0(0.51 \%)$ & $5094.2(1.13 \%)$ & $8316.4(1.42 \%)$ & $11700.2(1.33 \%)$ \\
\hline 9 & $2343.9(0.37 \%)$ & $5090.0(1.05 \%)$ & $8297.1(1.19 \%)$ & $11685.6(1.20 \%)$ \\
\hline By ABAQUS & 2335.2 & 5037.1 & 8199.7 & 11547.0 \\
\hline
\end{tabular}

Note. Relative error, ([prediction by the present model] - [result by ABAQUS])/[result by ABAQUS] $\times 100 \%$.

TABLE 2: Comparison of the nondimensional fundamental natural frequencies $(\bar{w})$ with various thickness ratios of the sandwich beam $(L / H=$ 5).

\begin{tabular}{|c|c|c|c|c|c|c|}
\hline \multirow{2}{*}{$p$} & \multicolumn{2}{|c|}{$1-1-1$} & \multicolumn{2}{|c|}{$1-2-1$} & \multicolumn{2}{|c|}{$1-8-1$} \\
\hline & Ref. $[*]$ & Present & Ref. [*] & Present & Ref. [*] & Present \\
\hline 0.5 & 7.3914 & $7.5552(2.22 \%)$ & 7.0539 & $7.0856(0.45 \%)$ & 6.3333 & $5.8824(-7.12 \%)$ \\
\hline 1 & 7.9726 & $8.0061(0.42 \%)$ & 7.5187 & $7.5140(-0.06 \%)$ & 6.6705 & $6.3057(-5.47 \%)$ \\
\hline 2 & 8.4655 & $8.3244(-1.67 \%)$ & 7.8960 & $7.8126(-1.06 \%)$ & 6.9233 & $6.6750(-3.59 \%)$ \\
\hline 5 & 8.9201 & $8.5768(-3.85 \%)$ & 8.2498 & $8.0356(-2.60 \%)$ & 7.1155 & $6.9648(-2.12 \%)$ \\
\hline 10 & 9.1193 & $8.6898(-4.71 \%)$ & 8.4162 & $8.1250(-3.46 \%)$ & 7.1884 & $7.0768(-1.55 \%)$ \\
\hline
\end{tabular}

Note. Ref. [*] $\equiv$ Vo et al. [17], and relative error: [(prediction by the present model) $-\left(\right.$ Ref. [*])]/(Ref. [*]) $\times 100 \% .\left(\bar{w}=\left(w L^{2} / H\right)\left(\rho_{c} / E_{c}\right)^{0.5}\right)$.

TABLE 3: Comparison of the nondimensional fundamental natural frequencies $(\bar{w})$ with various thickness ratios of the sandwich beam $(L / H=$ 20).

\begin{tabular}{lcccccc}
\hline$p$ & & $1-1-1$ & & $1-2-1$ & & $1-8-1$ \\
Present \\
\hline 0.5 & 10.2298 & $10.1771(-0.52 \%)$ & 9.7587 & $9.7449(-0.14 \%)$ & 8.2818 & $7.9373(-4.16 \%)$ \\
1 & 11.2767 & $11.0023(-2.43 \%)$ & 10.7706 & $10.6093(-1.50 \%)$ & 9.0064 & $8.6446(-4.02 \%)$ \\
2 & 11.9911 & $11.7095(-2.35 \%)$ & 11.5168 & $11.3559(-1.40 \%)$ & 9.6175 & $9.2733(-3.58 \%)$ \\
5 & 12.4199 & $12.2989(-0.97 \%)$ & 12.0423 & $11.9846(-0.48 \%)$ & 10.1297 & $9.8627(-2.64 \%)$ \\
10 & 12.5230 & $12.5740(0.41 \%)$ & 12.2122 & $12.2204(0.07 \%)$ & 10.3315 & $10.0985(-2.26 \%)$ \\
\hline
\end{tabular}

Note. Ref. [*] $\equiv$ Vo et al. [17], and relative error: [(prediction by the present model) $-($ Ref. [*])]/(Ref. $[*]) \times 100 \% .\left(\bar{w}=\left(w L^{2} / H\right)\left(\rho_{c} / E_{c}\right)^{0.5}\right)$.

aluminum foam and $\varphi$ is a coefficient chosen by experience. Simply for ease of accounting, $\varphi$ is taken to be 1.0.

Figure 3 shows fundamental frequency of the sandwich beams with the same thickness and different thickness ratio between face sheets and core. In Figure 3, legends 1-8-1, 17-1, 1-6-1, 1-5-1, 1-4-1, and 1-3-1 mean relative thickness of the sandwich beam following by top sheet, core, and bottom sheet. For example, 1-8-1 means the thickness of the core is eight times the thickness of face sheet.

When the gradient exponent $p$ is a constant, the fundamental frequency of the sandwich beam increases with the increasing density of the core firstly. And then the fundamental frequency arrives at a peak value. After that the fundamental frequency decreases slowly with the increasing density of the core. An interesting phenomenon is that the fundamental frequency is almost the same value (named the special frequency, $f_{\text {special }}$ for short) when the density of the core is a specific value ( $\rho_{\text {special }}$ for short) for all different thickness ratios of sandwich beams from 1-8-1 to 1-3-1. The reason is that the mass and stiffness of sandwich beams change and influence each other at the same time with changing thickness ratio. Figure 3 also shows that the special frequency $\left(f_{\text {special }}\right)$ presents an increasing trend when the gradient exponent changes from 0.1 to 10 .

Figure 4 shows that the fundamental frequency increases rapidly with increasing of the gradient exponent $p$ as $p$ is smaller than 1 for different densities of foam cores. The growth of the fundamental frequency slows down and it gradually reaches a constant value after the gradient exponent $p$ is larger than 1 . The reason is that the mass and the stiffness of the gradient face sheets do not change obviously after the gradient exponent $p$ is larger than 1 . 


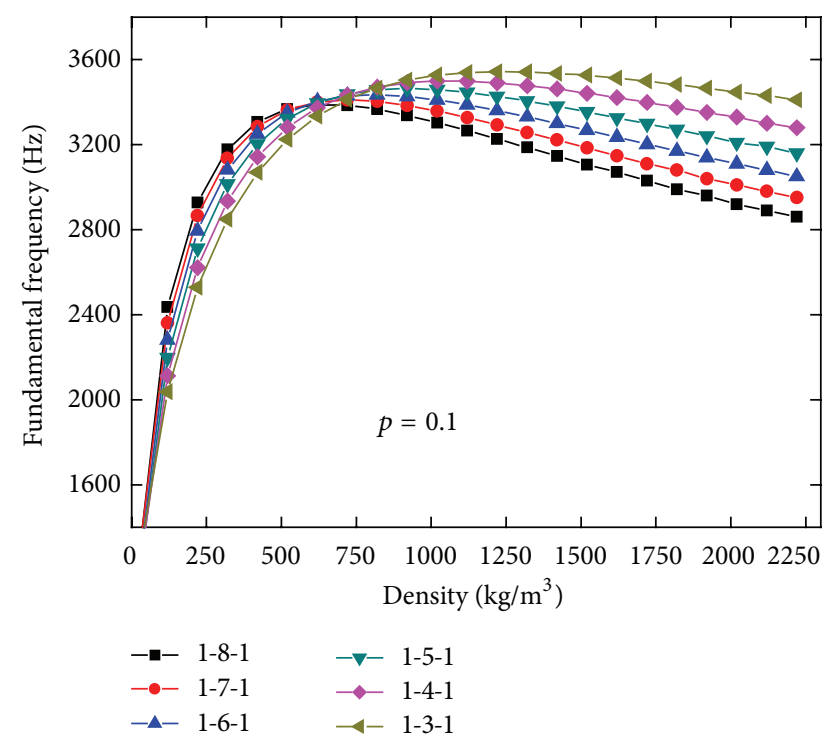

(a)

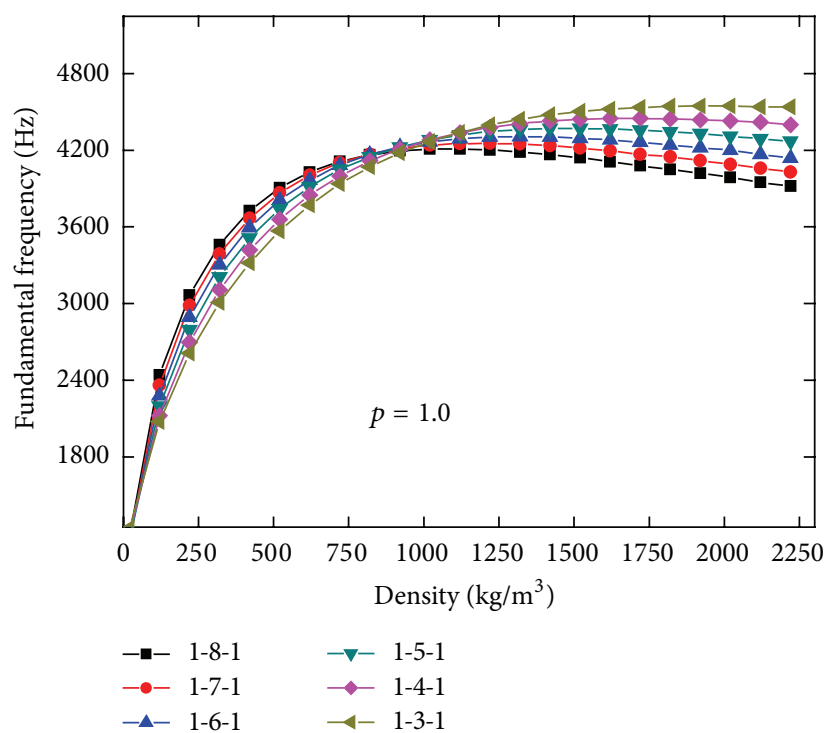

(b)

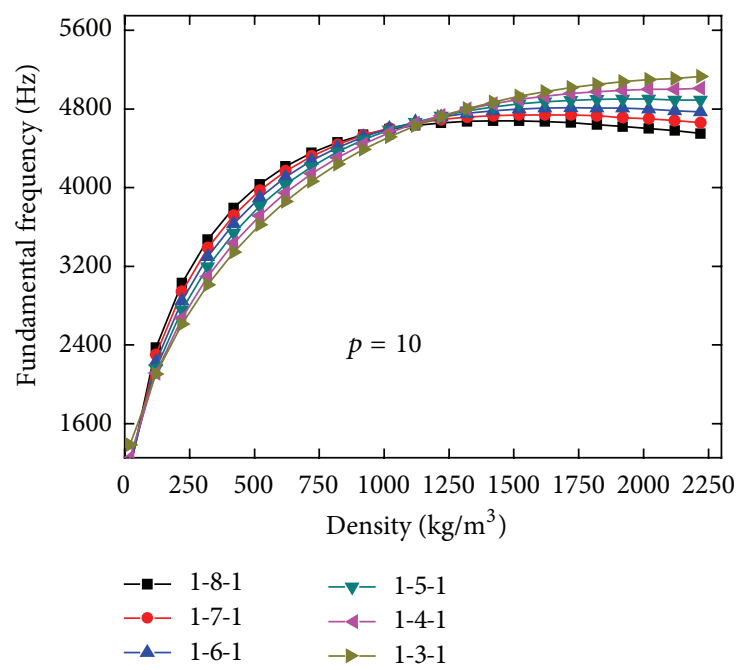

(c)

FiguRE 3: Fundamental frequency of the sandwich beams with different thickness ratios $\left(t^{f} / t^{c} / t^{f}\right)$ versus density of the core for different gradient exponents. (a) $p=0.1$; (b) $p=1.0$; (c) $p=10$.

\section{Conclusions}

(1) An analytical model to analyze fundamental frequency of sandwich beams with functionally graded face sheets and metallic foam core is developed.

(2) When the thickness $H$ of the sandwich beam is a constant and the thickness ratio of each part from bottom to top of the sandwich beam is varying, the fundamental frequency is very close to a fixed value ( $f_{\text {special }}$ for short) when the relative density of the core is taken to a specific value ( $\rho_{\text {special }}$ for short). This result can be used to optimize the design of the sandwich beams.

(3) The influences of the gradient exponent $p$ on the fundamental frequency of sandwich beams are very obvious. When the gradient exponent $p$ is larger than 1 , the influence is slight.

\section{Appendices}

\section{A. Stresses in Top and Bottom Face Sheets and Core}

Consider

$$
\begin{aligned}
& m_{0}^{f}=\int_{t^{c} / 2}^{t^{c} / 2+t^{f}} \rho^{f}(z) \mathrm{d} z=\int_{-t^{c} / 2-t^{f}}^{-t^{c} / 2} \rho^{f}(-z) \mathrm{d} z \\
& m_{1}^{f}=\int_{t^{c} / 2}^{t^{c} / 2+t^{f}} \rho^{f}(z)\left(z-\frac{t^{c}+t^{f}}{2}\right) \mathrm{d} z
\end{aligned}
$$




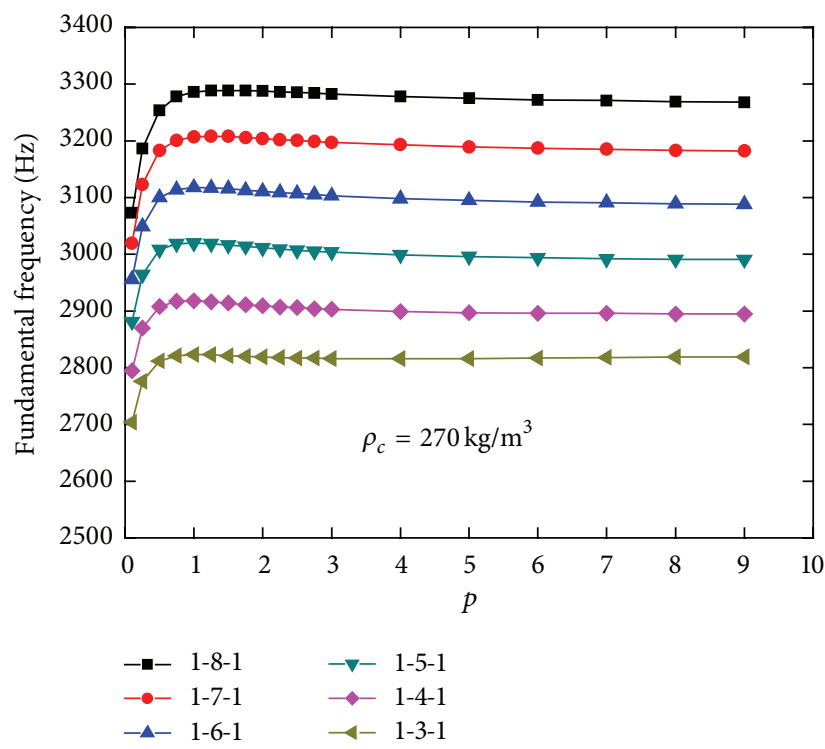

(a)

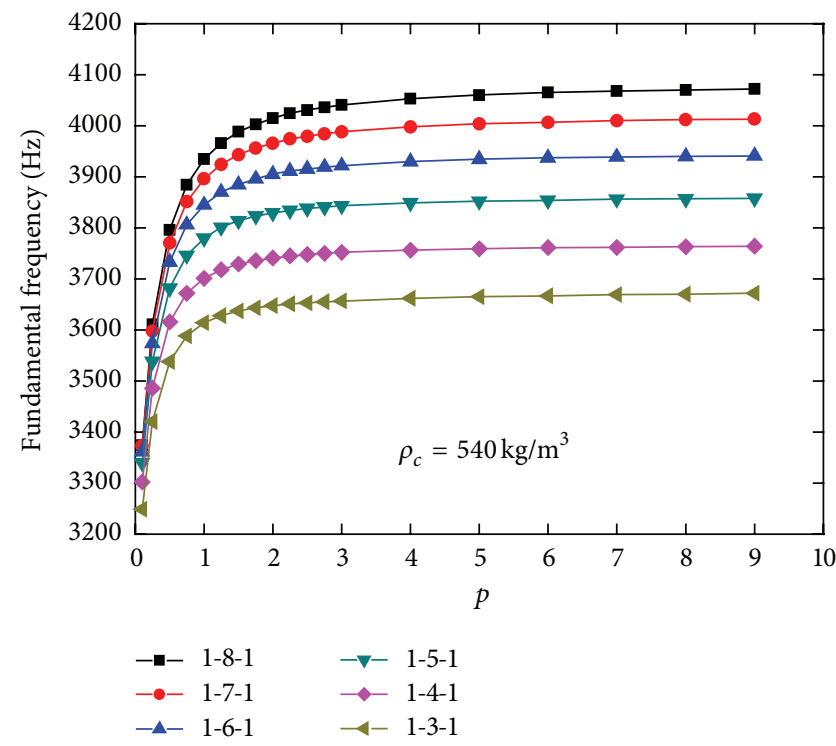

(b)

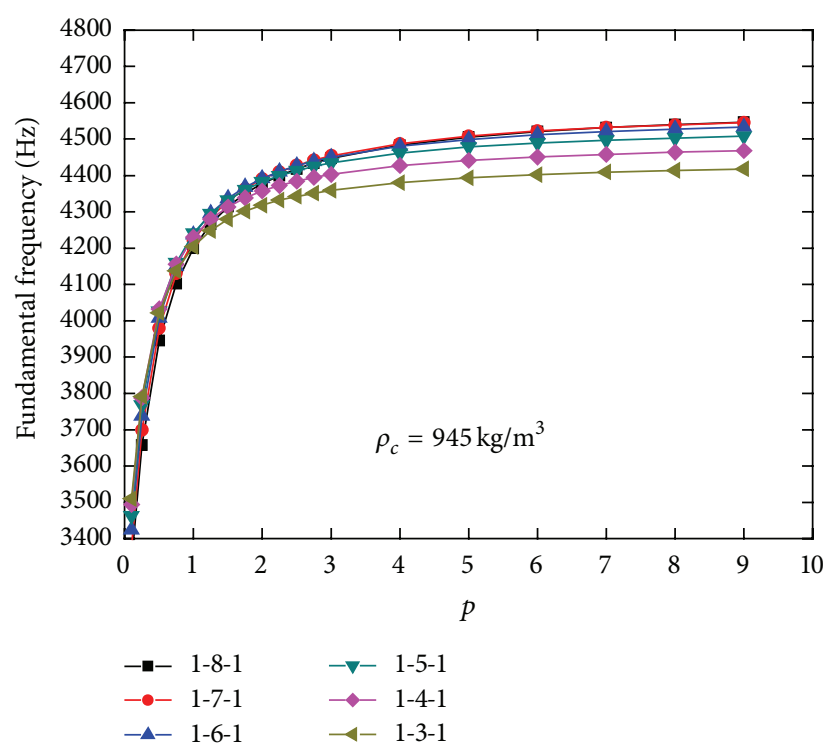

(c)

FIGURE 4: Fundamental frequency of the sandwich beams with different thickness ratios versus gradient exponent for different densities of foam core. (a) $\rho_{c}=270 \mathrm{~kg} / \mathrm{m}^{3}$; (b) $\rho_{c}=540 \mathrm{~kg} / \mathrm{m}^{3}$; (c) $\rho_{c}=945 \mathrm{~kg} / \mathrm{m}^{3}$.

$$
\begin{aligned}
& =\int_{-t^{c} / 2-t^{f}}^{-t^{c} / 2} \rho^{f}(-z)\left(z+\frac{t^{c}+t^{f}}{2}\right) \mathrm{d} z \\
m_{2}^{f} & =\int_{t^{c} / 2}^{t^{c} / 2+t^{f}} \rho^{f}(z)\left(z-\frac{t^{c}+t^{f}}{2}\right)^{2} \mathrm{~d} z \\
& =\int_{-t^{c} / 2-t^{f}}^{-t^{c} / 2} \rho^{f}(-z)\left(z+\frac{t^{c}+t^{f}}{2}\right)^{2} \mathrm{~d} z \\
m_{0}^{c} & =\rho_{c} t^{c} \\
m_{1}^{c} & =\frac{1}{3} \rho_{c} t^{c} .
\end{aligned}
$$

\section{B. Stiffness Quantities}

Consider

$$
\begin{aligned}
A_{1}^{t} & =A_{1}^{b}=A_{1}^{f}=\int_{(1 / 2) t^{c}}^{(1 / 2) t^{c}+t^{f}} E(z) \mathrm{d} z \\
& =\int_{-(1 / 2) t^{c}-t^{f}}^{-(1 / 2) t^{c}} E(-z) \mathrm{d} z \\
B_{1}^{t} & =B_{1}^{b}=B_{1}^{f}=\int_{(1 / 2) t^{c}}^{(1 / 2) t^{c}+t^{f}}\left(z-\frac{t^{c}+t^{f}}{2}\right) E(z) \mathrm{d} z
\end{aligned}
$$




$$
\begin{aligned}
& =\int_{-(1 / 2) t^{c}-t^{f}}^{-(1 / 2) t^{c}}\left(z+\frac{t^{c}+t^{f}}{2}\right) E(-z) \mathrm{d} z \\
D_{1}^{t} & =D_{1}^{b}=D_{1}^{f}=\int_{(1 / 2) t^{c}}^{(1 / 2) t^{c}+t^{f}}\left(z-\frac{t^{c}+t^{f}}{2}\right)^{2} E(z) \mathrm{d} z \\
& =\int_{-(1 / 2) t^{c}-t^{f}}^{-(1 / 2) t^{c}}\left(z+\frac{t^{c}+t^{f}}{2}\right)^{2} E(-z) \mathrm{d} z .
\end{aligned}
$$

\section{Elements in Mass Matrix and Stiffness Matrix}

Consider

$$
\begin{aligned}
& K_{11}=\int_{0}^{l} 2 A_{1}^{f}\left\{\phi_{1 i}^{a}\right\}^{T}\left\{\phi_{1 i, 11}^{a}\right\} \mathrm{d} x \\
& K_{13}=-\int_{0}^{l} 2 B_{1}^{f}\left\{\phi_{1 i}^{a}\right\}^{T}\left\{\phi_{3 i, 111}^{a}\right\} \mathrm{d} x \\
& M_{11}=-2 m_{0}^{f} \int_{0}^{l}\left\{\phi_{1 i}^{a}\right\}^{T}\left\{\phi_{1 i}^{a}\right\} \mathrm{d} x \\
& M_{13}=2 m_{1}^{f} \int_{0}^{l}\left\{\phi_{1 i}^{a}\right\}^{T}\left\{\phi_{3 i, 1}^{a}\right\} \mathrm{d} x \\
& K_{22}=\int_{0}^{l}\left\{\phi_{1 i}^{d}\right\}^{T}\left(2 A_{1}^{f}\left\{\phi_{1 i, 11}^{d}\right\}-\frac{4 G^{c}}{t^{c}}\left\{\phi_{1 i}^{d}\right\}\right) \mathrm{d} x \\
& K_{23}=-\frac{2\left(t^{c}+t^{f}\right)}{t^{c}} G^{c} \int_{0}^{l}\left\{\phi_{1 i}^{d}\right\}^{T}\left\{\phi_{3 i, 1}^{a}\right\} \mathrm{d} x \\
& K_{24}=-\int_{0}^{l} 2 B_{1}^{f}\left\{\phi_{1 i}^{d}\right\}^{T}\left\{\phi_{3 i, 111}^{d}\right\} \mathrm{d} x \\
& M_{22}=-2 m_{0}^{f} \int_{0}^{l}\left\{\phi_{1 i}^{d}\right\}^{T}\left\{\phi_{1 i}^{d}\right\} \mathrm{d} x \\
& M_{24}=2 m_{1}^{f} \int_{0}^{l}\left\{\phi_{1 i}^{d}\right\}^{T}\left\{\phi_{3 i, 1}^{d}\right\} \mathrm{d} x \\
& M_{31}=-2 m_{1}^{f} \int_{0}^{l}\left\{\phi_{3 i}^{a}\right\}^{T}\left\{\phi_{1 i, 1}^{a}\right\} \mathrm{d} x \\
& M_{33}=\int_{0}^{l}\left\{\phi_{3 i}^{a}\right\}^{T}\left(-2 m_{0}^{f}\left\{\phi_{3 i}^{a}\right\}+2 m_{2}^{f}\left\{\phi_{3 i, 11}^{a}\right\}\right. \\
& \left.-m_{0}^{c}\left\{\phi_{3 i}^{a}\right\}\right) \mathrm{d} x \\
& K_{31}=\int_{0}^{l} 2 B_{1}^{f}\left\{\phi_{3 i}^{a}\right\}^{T}\left\{\phi_{1 i, 111}^{a}\right\} \mathrm{d} x \\
& K_{32}=\frac{2\left(t^{c}+t^{f}\right)}{t^{c}} G^{c} \int_{0}^{l}\left\{\phi_{3 i}^{a}\right\}^{T}\left\{\phi_{1 i, 1}^{d}\right\} \mathrm{d} x \\
& K_{33}=\int_{0}^{l}\left\{\phi_{3 i}^{a}\right\}^{T}\left[-2 D_{1}^{f}\left\{\phi_{3 i, 1111}^{a}\right\}+\frac{\left(t^{f}+t^{c}\right)^{2}}{t^{c}} \cdot G^{c}\right. \\
& \left.\cdot\left\{\phi_{3 i, 11}^{a}\right\}\right] \mathrm{d} x
\end{aligned}
$$

$$
\begin{aligned}
M_{42} & =-2 m_{1}^{f} \int_{0}^{l}\left\{\phi_{3 i}^{d}\right\}^{T}\left\{\phi_{1 i, 1}^{d}\right\} \mathrm{d} x \\
M_{44} & =\int_{0}^{l}\left\{\phi_{3 i}^{d}\right\}^{T}\left(-2 m_{0}^{f}\left\{\phi_{3 i}^{d}\right\}+2 m_{2}^{f}\left\{\phi_{3 i, 11}^{d}\right\}\right. \\
& \left.-m_{1}^{c}\left\{\phi_{3 i}^{d}\right\}\right) \mathrm{d} x \\
K_{42} & =\int_{0}^{l} 2 B_{1}^{f}\left\{\phi_{3 i}^{a}\right\}^{T}\left\{\phi_{1 i, 111}^{d}\right\} \mathrm{d} x \\
K_{44} & =\int_{0}^{l}\left\{\phi_{3 i}^{d}\right\}^{T}\left[-2 D_{1}^{f}\left\{\phi_{3 i, 1111}^{d}\right\}-\frac{4 E^{c}}{t^{c}}\left\{\phi_{3 i}^{d}\right\}\right] \mathrm{d} x \\
f_{3}= & -q_{3}^{t} \int_{0}^{l}\left\{\phi_{3 i}^{a}\right\} \mathrm{d} x \\
f_{4}= & -q_{3}^{t} \int_{0}^{l}\left\{\phi_{3 i}^{d}\right\} \mathrm{d} x .
\end{aligned}
$$

\section{Conflict of Interests}

The authors declare that there is no conflict of interests regarding the publication of this paper.

\section{Acknowledgment}

This work is supported by the National Natural Science Foundation of China (11372237).

\section{References}

[1] M. Koizumi, “The concept of FGM," Ceramic Transactions of Functional Graded Materials, vol. 34, pp. 3-10, 1993.

[2] D. K. Jha, T. Kant, and R. K. Singh, "Free vibration response of functionally graded thick plates with shear and normal deformations effects," Composite Structures, vol. 96, pp. 799823, 2013.

[3] S. Xiang, G.-W. Kang, M.-S. Yang, and Y. Zhao, "Natural frequencies of sandwich plate with functionally graded face and homogeneous core," Composite Structures, vol. 96, pp. 226-231, 2013.

[4] A. M. Zenkour, "A comprehensive analysis of functionally graded sandwich plates: part 1-deflection and stresses," International Journal of Solids and Structures, vol. 42, no. 18-19, pp. 5224-5242, 2005.

[5] A. M. Zenkour, "A comprehensive analysis of functionally graded sandwich plates. Part 2. Buckling and free vibration," International Journal of Solids and Structures, vol. 42, no. 18-19, pp. 5243-5258, 2005.

[6] B. Woodward and M. Kashtalyan, "Bending response of sandwich panels with graded core: 3D elasticity analysis," Mechanics of Advanced Materials and Structures, vol. 17, no. 8, pp. 586-594, 2010.

[7] A. M. Zenkour and N. A. Alghamdi, "Bending analysis of functionally graded sandwich plates under the effect of mechanical and thermal loads," Mechanics of Advanced Materials and Structures, vol. 17, no. 6, pp. 419-432, 2010. 
[8] M. Cinefra and M. Soave, "Accurate vibration analysis of multilayered plates made of functionally graded materials," Mechanics of Advanced Materials and Structures, vol. 18, no. 1, pp. 3-13, 2011.

[9] M. Kashtalyan and M. Menshykova, "Three-dimensional elasticity solution for sandwich panels with a functionally graded core," Composite Structures, vol. 87, no. 1, pp. 36-43, 2009.

[10] Q. Li, V. P. Iu, and K. P. Kou, "Three-dimensional vibration analysis of functionally graded material sandwich plates," Journal of Sound and Vibration, vol. 311, no. 1-2, pp. 498-515, 2008.

[11] A. M. A. Neves, A. J. M. Ferreira, E. Carrera et al., "Static, free vibration and buckling analysis of isotropic and sandwich functionally graded plates using a quasi-3D higher-order shear deformation theory and a meshless technique," Composites Part B: Engineering, vol. 44, no. 1, pp. 657-674, 2013.

[12] A. M. A. Neves, A. J. M. Ferreira, E. Carrera et al., "Free vibration analysis of functionally graded shells by a higher-order shear deformation theory and radial basis functions collocation, accounting for through-the-thickness deformations," European Journal of Mechanics A: Solids, vol. 37, pp. 24-34, 2013.

[13] E. Carrera, "Evaluation of layer-wise mixed theories for laminated plates analysis," AIAA Journal, vol. 36, no. 5, pp. 830-839, 1998.

[14] E. Carrera, S. Brischetto, M. Cinefra, and M. Soave, "Effects of thickness stretching in functionally graded plates and shells," Composites Part B: Engineering, vol. 42, no. 2, pp. 123-133, 2011.

[15] A. J. M. Ferreira, C. M. C. Roque, and P. A. L. S. Martins, "Analysis of composite plates using higher-order shear deformation theory and a finite point formulation based on the multiquadric radial basis function method," Composites Part B: Engineering, vol. 34, no. 7, pp. 627-636, 2003.

[16] H.-T. Thai, T.-K. Nguyen, T. P. Vo, and J. Lee, "Analysis of functionally graded sandwich plates using a new first-order shear deformation theory," European Journal of MechanicsA/Solids, vol. 45, pp. 211-225, 2014.

[17] T. P. Vo, H.-T. Thai, T.-K. Nguyen, A. Maheri, and J. Lee, "Finite element model for vibration and buckling of functionally graded sandwich beams based on a refined shear deformation theory," Engineering Structures, vol. 64, pp. 12-22, 2014.

[18] J. Hohe and L. Librescu, "Recent results on the effect of the transverse core compressibility on the static and dynamic response of sandwich structures," Composites Part B: Engineering, vol. 39, no. 1, pp. 108-119, 2008.

[19] L. Mu, B. He, D. Xiao, G. Zhao, and C. Cho, "Dynamic response of beam-like composite sandwich plates with transversely flexible cores," Ocean Engineering, vol. 108, pp. 778-788, 2015.

[20] Z. Qin and R. C. Batra, "Local slamming impact of sandwich composite hulls," International Journal of Solids and Structures, vol. 46, no. 10, pp. 2011-2035, 2009.

[21] M. F. Ashby, Metal Foams: A Design Guide, ButterworthHeinemann, Boston, Mass, USA, 2000. 


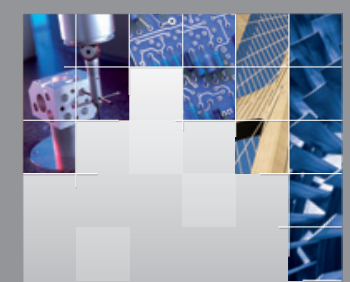

\section{Enfincering}
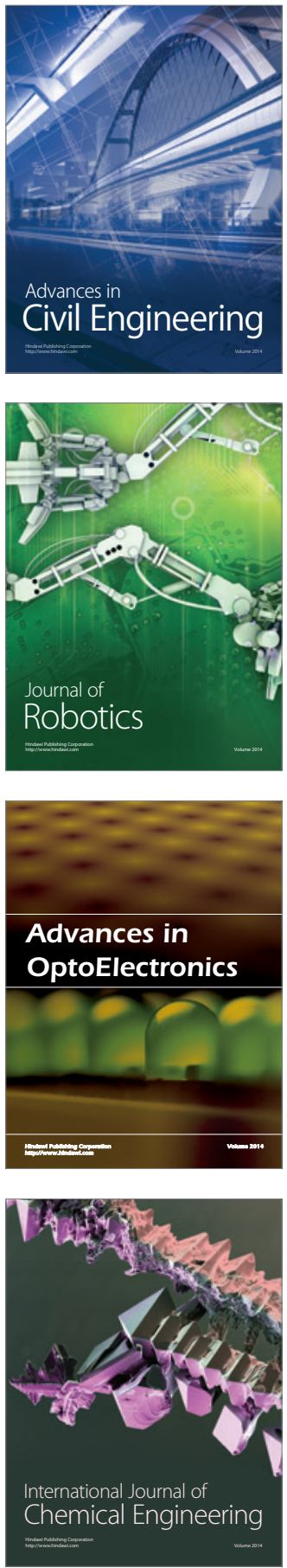

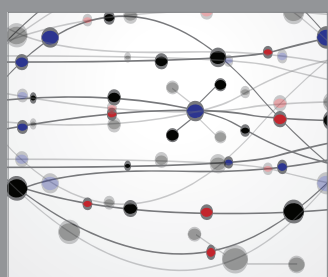

The Scientific World Journal

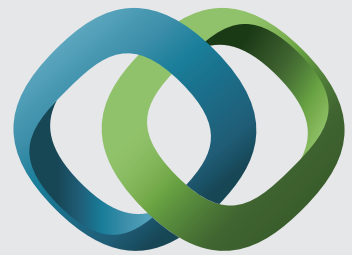

\section{Hindawi}

Submit your manuscripts at

http://www.hindawi.com
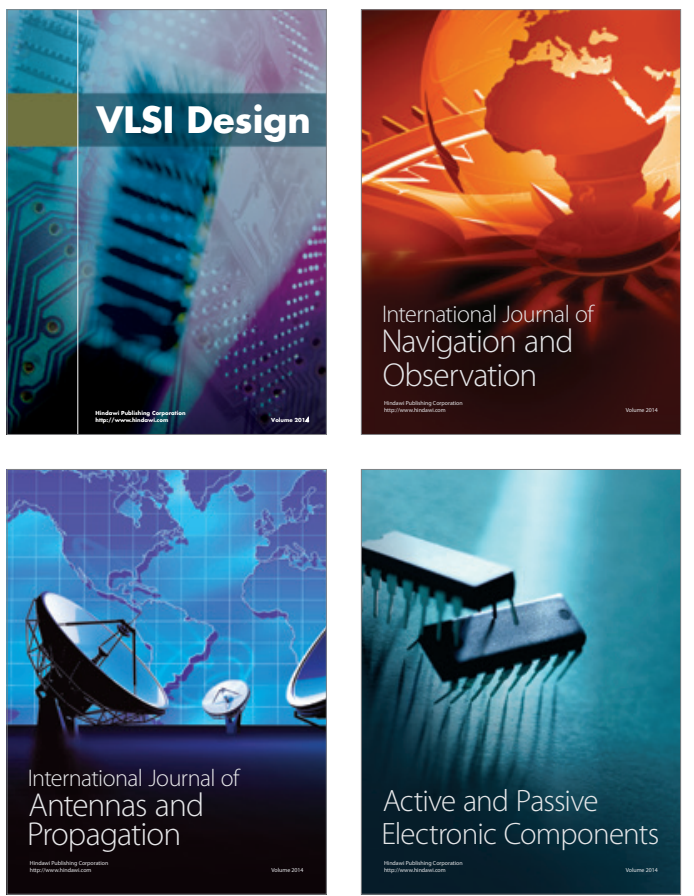
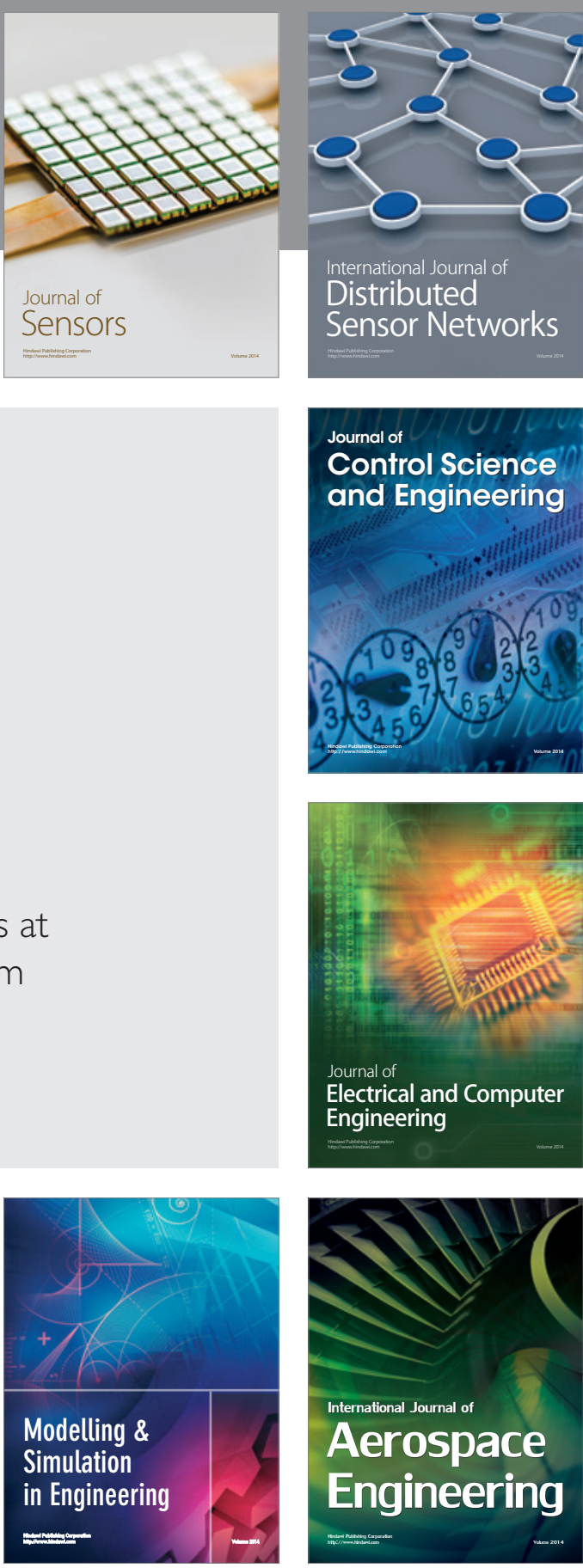

International Journal of

Distributed

Sensor Networks

Journal of

Control Science

and Engineering
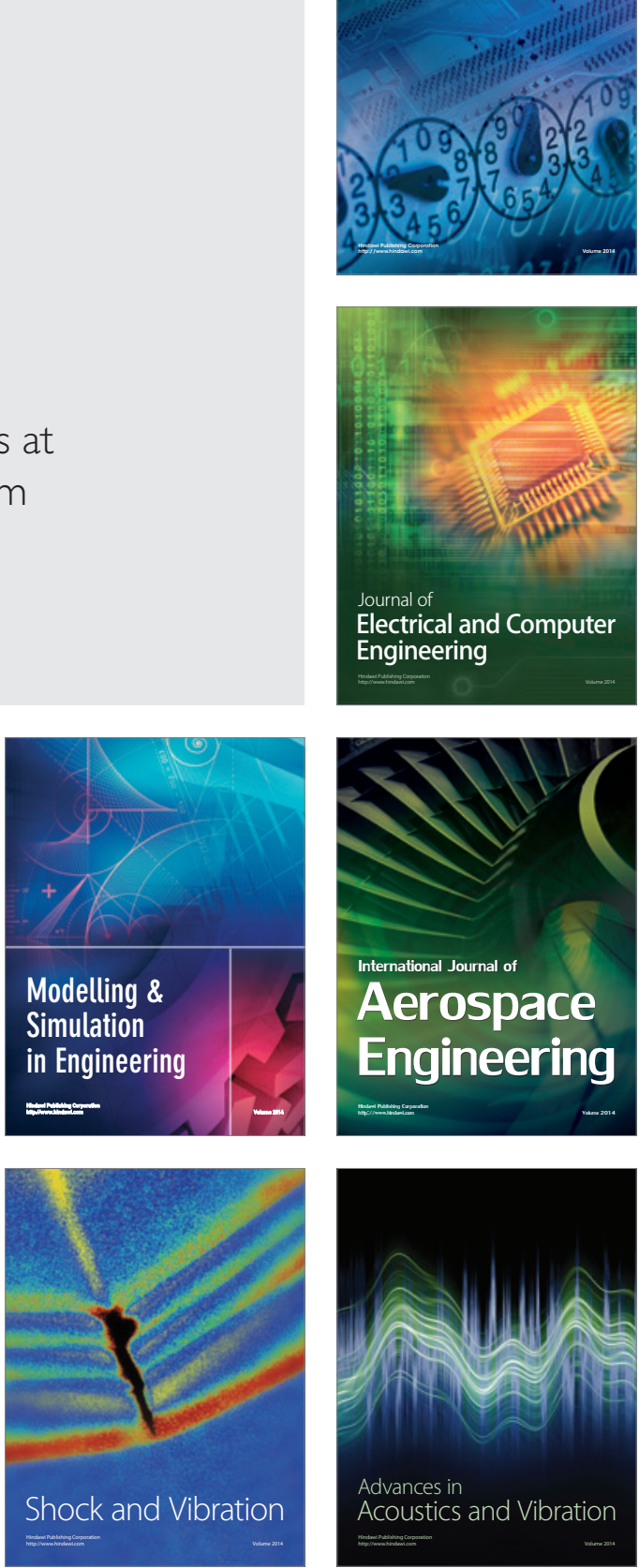